\title{
Extracellular Vesicles in Autologous Cell Salvaged Blood in Orthopedic Surgery
}

\author{
Maximilian Kutschera ${ }^{1}$, Agnes Pischlaeger ${ }^{2}$, Larissa Sztulman ${ }^{2}$, Sibylle Kietaibl ${ }^{1,3, *}$ and Andreas Spittler ${ }^{2,4}{ }^{1}$ \\ 1 Campus Prater, Sigmund Freud University, 1020 Vienna, Austria; max.kutschera@gmail.com \\ 2 Research Lab, Department of Surgery, Medical University of Vienna, 1090 Vienna, Austria; \\ agnes.pischlaeger@gmail.com (A.P.); larissa.sztulman@gmail.com (L.S.); \\ andreas.spittler@meduniwien.ac.at (A.S.) \\ 3 Austria Evangelical Hospital Vienna, 1180 Vienna, Austria \\ 4 Core Facility Flow Cytometry, Medical University of Vienna, 1090 Vienna, Austria \\ * Correspondence: s.kietaibl@ekhwien.at; Tel.: +43-1-40422-4040
}

Citation: Kutschera, M.; Pischlaeger, A.; Sztulman, L.; Kietaibl, S.; Spittler, A. Extracellular Vesicles in Autologous Cell Salvaged Blood in Orthopedic Surgery. Surgeries 2021, 2 , 84-91. https://doi.org/10.3390/ surgeries2010007

Academic Editor: Nicola Maffulli

Received: 4 January 2021

Accepted: 4 February 2021

Published: 8 February 2021

Publisher's Note: MDPI stays neutral with regard to jurisdictional claims in published maps and institutional affiliations.

Copyright: (c) 2021 by the authors. Licensee MDPI, Basel, Switzerland. This article is an open access article distributed under the terms and conditions of the Creative Commons Attribution (CC BY) license (https:// creativecommons.org/licenses/by/ $4.0 /)$.

\begin{abstract}
Background: Cell salvage is highly recommended in orthopedic surgery to avoid allogeneic transfusions. Preparational steps during cell salvage may induce extracellular vesicle (EV) formation with potential thrombogenic activity. The purpose of our study was to assess the appearance of EVs at retransfusion. (2) Methods: After ethics committee approval and informed consent, blood was withdrawn from the autotransfusion system (Xtra, Sorin, Germany) of 23 patients undergoing joint arthroplasty. EVs were assessed by flow cytometry in two times centrifugated samples. EVs were stained with specific antibodies against cellular origins from platelets (CD41), myeloid cells (CD15), monocytes (CD14), and erythrocytes (CD235a). The measured events/ $\mu \mathrm{L}$ in the flow cytometer were corrected to the number of EVs in the retransfusate. (3) Results: We measured low event rates of EVs from platelets and myeloid origin $(<1$ event $/ \mu \mathrm{L})$ and from monocytic origin ( $<2$ events $/ \mu \mathrm{L}$ ). Mean event rates of 17,042 events/ $\mu \mathrm{L}$ (range 12-81,164 events/ $\mu \mathrm{L}$ ) were found for EVs from red blood cells. (4) Conclusion: Retransfusate contains negligible amounts of potentially thrombogenic EVs from platelet and monocytic origin. Frequent EVs from erythrocytes may indicate red blood cell destruction and/or activation during autologous cell salvage. Further research is needed to investigate the clinical relevance of EVs from salvaged red blood cells.
\end{abstract}

Keywords: cell salvage; extracellular vesicle; microparticle

\section{Introduction}

Major surgery may lead to severe bleeding, anemia, hypovolemia, and allogeneic transfusion requirements [1]. Autologous cell salvage is an important clinical tool in perioperative patient blood management (PBM) to decrease loss of blood and anemia [1]. Randomized controlled trials and meta-analyses further confirm that autologous cell salvage reduces the need for perioperative allogeneic red blood cell (RBC) transfusions [2-5]. Guidelines from the European Society of Anesthesiology highly recommended the use of cell salvage, which is helpful for blood conservation in major cardiac and orthopedic surgery (GRADE 1B) [1].

Allogeneic RBC preparation involves centrifugation and dissolution; eventual cell damage and/or activation result in extracellular vesicle (EV) formation [6]. The amount of EVs increases upon storage time as part of the RBC storage lesion [6]. This phenomenon has been linked to transfusion-associated thromboembolic and inflammatory complications $[7,8]$. EVs in autotransfusion concentrates prior to retransfusion remain poorly understood. Similar to allogeneic RBC preparation, also autologous cell salvage involves centrifugation and washing procedures which may activate and/or destroy the patient's blood cells, resulting in the generation of cell-derived EVs. Considering the increasing clinical use of cell salvage and innovations in cell salvage technology, markers 
for cell damage can be helpful in defining quality and safety of retransfused blood. Accordingly, we assessed extracellular vesicles from autologous platelets (CD41), monocytes (CD14) and myeloid cell origin (CD15), and erythrocytes (CD235a) using flow cytometry in a last-generation cell saver during orthopedic surgery.

\section{Materials and Methods}

Ethics committee approval for this study was provided by the Ethical Committee of Sigmund Freud Private University Vienna, Vienna, Austria (Ethical Committee Number 002/2018) (Vicechairperson G. Endler) on 5 June 2018. Informed consent was obtained from 23 adult patients scheduled for major orthopedic surgery. Inclusion criteria were primary hip or knee joint replacement with routine use of autologous cell salvage. Exclusion criteria were pregnancy, preexisting hematological, or bleeding disorders.

Cell salvage was performed according to our hospital-internal standard operating procedures. The general method of autologous wound blood salvage has been summarized elsewhere [9]. We used the Xtra (Sorin, Germany) with standard equipment. Blood is collected from the wound, dissolved in an anticoagulated crystalloidal solution (unfractionated heparin $25.000 \mathrm{IU}$ per $1 \mathrm{~L}$ sodium chloride $0.9 \%$ ), and centrifugated at $820-1480 \times \mathrm{g}$. Performance data of the Xtra system: hematocrit of $54 \%$, heparin removal of $99.7 \%$, potassium removal of $95 \%$, free hemoglobin removal of $97 \%$ [9].

In our study, cell salvage was used during and after surgery in the recovery room for a maximum of $6 \mathrm{~h}$ after initiation. At that time point, patients received their retransfusion. According to PBM, the indication for retransfusion was salvage of patient's own blood after major orthopedic surgery [1]. Salvaged erythrocytes were retransfused via a peripheral venous line after passing a 170-200 $\mu \mathrm{m}$ filter. From the remnant blood in lines and filter $3.5 \mathrm{~mL}$ of cell salvaged blood concentrate was withdrawn by dripping into a collection cuvette with sodium citrate (BD Vacutainer, Becton Dickinson, France).

Flow cytometric analysis was performed using the CytoFLEX LX ${ }^{\mathrm{TM}}$ flow cytometer (Beckman Coulter, Brea, CA, USA). To avoid swarm effects from the concentrated EVs, if necessary autologous cell salvage blood was diluted as described previously [10].

Detection of blood cell derived EVs was facilitated by means of lactadherin (AF647 labelled, CellSystems / Biotechnology, Troisdorf, Germany) labeling, which detects phosphatidylserine (PS) translocated from the inner to the outer membrane leaflet and is present on at least a subpopulation of EVs [11]. To identify EVs which are PS negative, additional staining using calcein AM (Calcein Green, ThermoFisher Scientific, Waltham, MA, USA) was performed. Calcein AM is taken up from EVs and converted by esterases to Calcein. Calcein can be excited by $488 \mathrm{~nm}$ laser light and emits light in a range of around $515 \mathrm{~nm}$ wavelength. Lactadherin in combination together with calcein served as trigger parameters to further identify EVs from different cell sources. The following monoclonal antibodies were used to describe subsets of extracellular vesicles: Anti-CD41 (PE-Cy7, Beckman Coulter, Brea, CA, USA) mAb against platelets origin, anti-CD14 mAb (PE-Cy7, Biolegend, San Diego, CA, USA) against monocyte origin, CD15 (PE-Cy7, Biolegend, San Diego, CA, USA) against myeloid origin and CD235a (PE, Beckman Coulter, Brea, CA, USA) against red blood cell origin $[10,12]$. To avoid the detection of antibody aggregates by flow cytometry, antibodies were centrifuged before the staining procedure at 17,000 $\mathrm{g}$ for $10 \mathrm{~min}$. The optimal concentration of antibodies was preliminary determined in antibody titration experiments. For staining of EVs a daily stock solution of centrifuged antibodies was prepared. Samples were pre-diluted in phosphate-buffered salt solution (PBS) and stained on ice with an aliquot of the antibodies stock solution for $1.5 \mathrm{~h}$. Afterwards EVs were incubated with lactadherin and calcein AM for additional $30 \mathrm{~min}$ on ice and fixed with paraformaldehyde to minimize the staining of free plasma esterases. The stained samples were then measured by flow cytometry within $1.5 \mathrm{~h}$. As depicted in Figure 1A, $1 \mu \mathrm{m}$ size silica beads were first measured using the VSSC as a trigger channel to estimate the approximate size of the measured EVs. A detergent-lysed plasma sample stained with isotype antibodies was then used as a negative control from the pregated EV population 
(Figure 1B,C). In order to detect particles labeled with antibodies, a combination of trigger channels was set to calcein or lactadherin, depending on which signal gives a positive signal first. The entirety of the lactadherin and/or calcein positive signals were then used for further analysis of the specific antibody stains. The results are given in events $/ \mu \mathrm{L}$ and show the concentration in the salvaged blood sample calculated from the dilution factor of the measured sample.

A

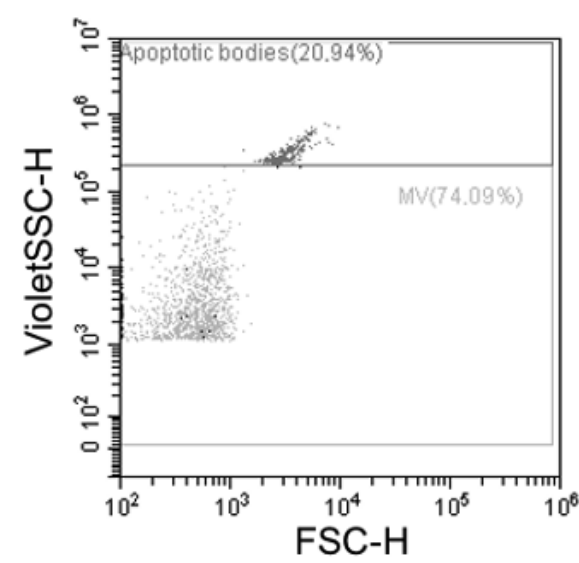

C

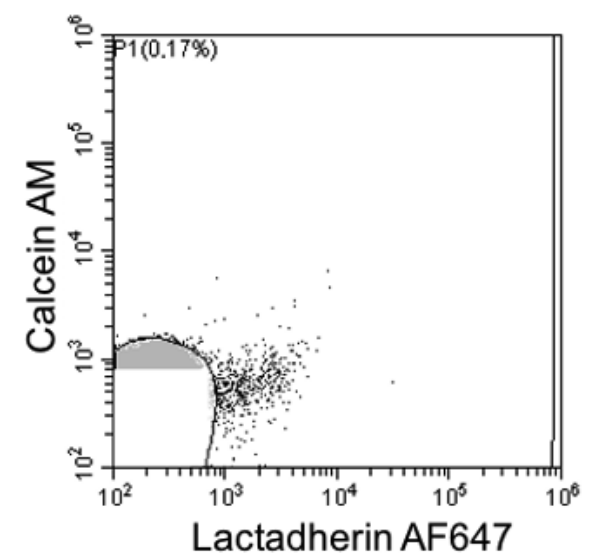

B

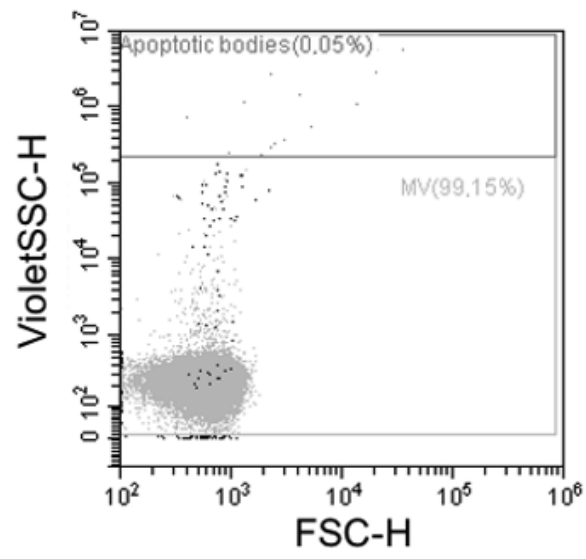

D

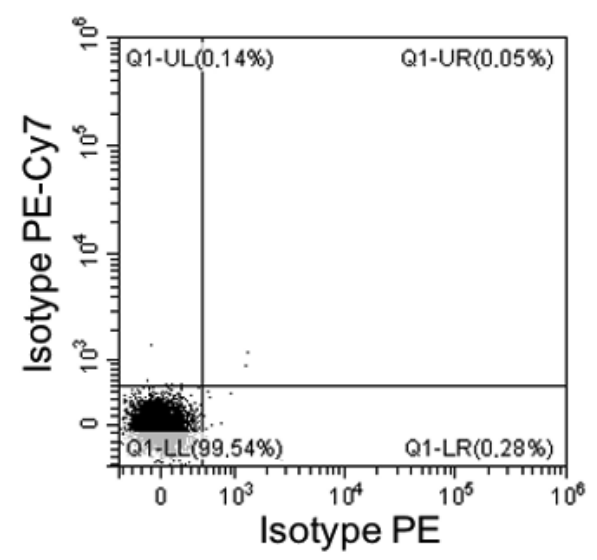

Figure 1. In a first step, silica beads in a size of $1 \mu \mathrm{m}$ were measured using the VioletSSC as the triggering signal. Below the $1 \mu \mathrm{m}$ bead population, a gate was set called Microvesicles (MV, A). Afterwards the detergent lysed control sample incubated with calcein AM (FITC) and stained with lactadherin, labeled with AF647, as well as with isotype antibodies, labeled with PE or PE-Cy7, were measured. Panel (B) shows the scattergram of calcein AM or lactadherin AF647 triggered control sample (C). A gate (P1) was drawn around the edges of the negative calcein/lactadherin sample and transferred to the isotype dotplot (D), where the lower left quadrant regions define the borders of the negative population.

Demographic data of patients, amount of autologous blood retransfused, allogeneic $\mathrm{RBC}$ requirements, and any complication including postoperative thrombosis, re-bleeding, and inflammation until hospital discharge were documented.

Statistics: Presented results are descriptive.

\section{Results}

\subsection{Clinical Data}

Twelve male and 11 female patients had a mean age $74 \pm 9$ years. The amount of retransfused $\mathrm{RBC}$ concentrate was $134 \pm 45 \mathrm{~mL}$. Perioperative clinical course of patients 
was uneventful, no allogeneic transfusions were required, no thromboembolic events and no infections were documented.

\subsection{Flow Cytometry Data}

After lactadherin-, calcein-, and blood cell-specific labeling EVs were detected. We measured low event rates of EVs from platelets, from myeloid and from monocytic origin (Table 1). High numbers of EVs from red blood cell origin were present in the concentrated salvaged sample (Table 1). The amount of antibody-positive microparticles is summarized in Table 1.

Table 1. Events $/ \mu \mathrm{L}$ in autologous cell salvage (retransfusate) from calcein /lactadherin gated particles.

\begin{tabular}{ccc}
\hline Antigen & Origin & Events/ $\mu$ L Mean (Range) \\
\hline CD235a & erythrocytes & $17,042(12-81,164)$ \\
CD14 & monocytes & $<2$ \\
CD15 & myelocytes & $<2$ \\
CD41 & platelets & $<1$ \\
\hline
\end{tabular}

Control samples are shown in Figure 1.

A typical pattern of labeling is shown in Figure 2.

A

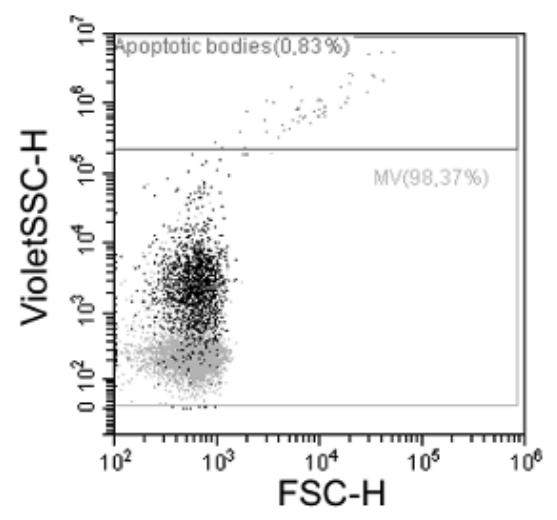

C

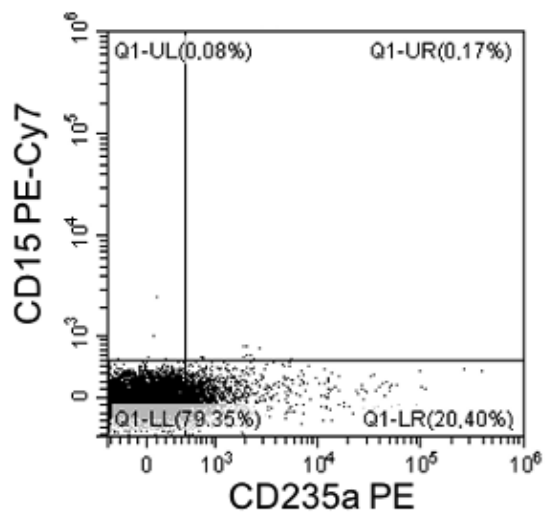

B

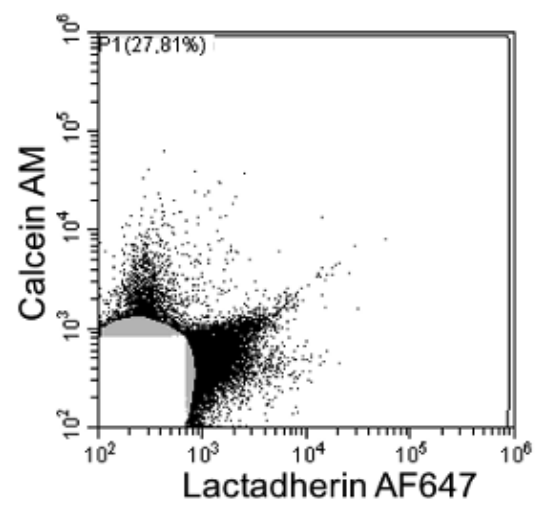

D

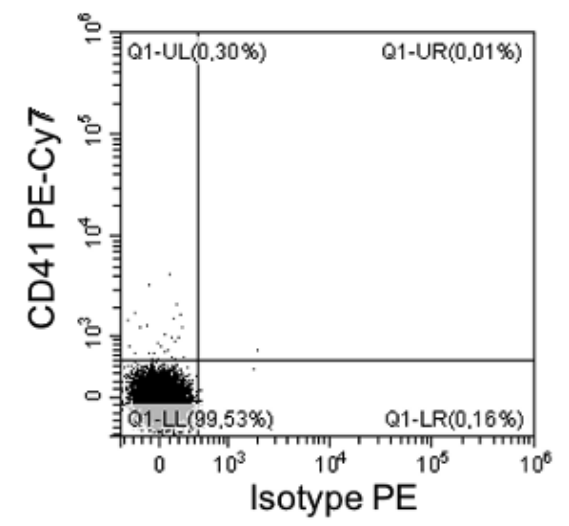

E

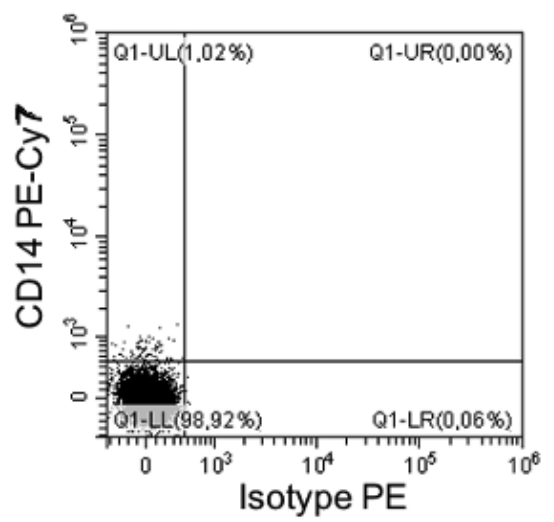

Figure 2. Scatterplot (A) shows the events from calcein AM or lactadherin AF647 triggered sample (B). Three samples from the same plasma were stained either with the combination of anti-CD15 PE-Cy7 and anti-CD235a PE antibodies (C), or with anti-CD41 PE-Cy7 and isotype PE-labeled control antibodies (D), or with anti-CD14 PE-Cy7 and isotype PE-labeled control antibodies (E). Positive events/ $\mu \mathrm{L}$ in quadrants QI1-UL and in Q1-LR were calculated by the CytoFLEX LX and presented in the statistics. 


\section{Discussion}

Our study describes extracellular vesicles (EVs) of human platelets, of myeloid and monocytic cell origin, as well as erythrocytes after autologous cell salvage in orthopedic surgery demonstrating that recovered salvaged blood is not free of cell-derived biological material (Figure 1). The total number of EVs from myeloid, monocytic, and platelet origin is very low (Table 1). Conventional flow cytometry cannot even detect such microparticles by using forward and side scatter patterns only. Characteristics of these EVs are their small size of $0.1-1 \mu \mathrm{m}$, lack of a nucleolus and synthetic capacity, exhibition of negatively-charged phospholipids in their membrane. In our experiments, condensing preparational steps before flow cytometric analysis involving centrifugation and fluorochrome-labeling were required for counting such rare occurrence rates of microparticles. The finding of low event rates of myeloid, monocytic, and platelet EVs is reconfirming for clinicians as a safety marker of autologous cell salvage. Our findings show no safety signal in terms of exaggerated microparticle load and support the use of fresh autologous salvaged blood in major orthopedic surgery. Research is ongoing on the (patho)physiological role of such EVs. By carrying phospholipids and tissue factor, EVs can promote hemostasis, specifically by increasing platelet adhesion, platelet aggregation, and thrombin generation [13-15]. Patients with deficient microparticle-mediated coagulation resemble deficiency of factor XI (hemophilia C) [16]. Similarly, microparticles may prevent excessive perioperative bleeding. Several studies point to an inflammatory potential of microparticles [17]. In pericardial blood, microparticles activate the complement system [18]. Considering the findings of the present study, it seems very unlikely that retransfused EVs in salvaged blood exert a clinically relevant procoagulant, anticoagulant, or inflammatory activity because of the dilution of very low numbers of EVs in the patients circulation and prompt macrophagic elimination. We observed in our small patient cohort no thromboembolic events, no rebleeding, and no infections after orthopedic surgery and autologous blood retransfusion. Our patients did not require any allogeneic blood transfusion. All patients received routine thromboprophylaxis according to the recommendations from the European Society of Anesthesiology [19].

Despite differences in methodology of flow cytometry, our non-red blood cell EV counts from platelets and monocytes appear markedly lower compared to cell salvage from a first-generation cell saver machine CATS (Fresenius AG, Bad Homburg, Germany) [20]. Differences in centrifugation forces may account for this discrepancy [21-23]. Other quality criteria including elimination rate of leukocytes (35-74\% in CATS, $95-99 \%$ in Xtra) and platelets (80-90\% in CATS, $95-99 \%$ in Xtra) document improved performance of the lastgeneration cell saver.

In our experiments the concentration of EVs produced from red blood cells was high (Table 1). This finding may indicate that during preparational steps of cell salvage erythrocytes may get destroyed and/or activated, resulting in the generation of red cell-derived EVs. Manufacturing methods have been described to determine EVs in allogeneic RBC preparation $[24,25]$. The (patho)physiological role of erythrocyte EVs is poorly understood. In critically ill patients, erythrocyte-derived microparticles had a microcirculatory impact $[26,27]$. EVs isolated from the plasma of animals with sepsis significantly decreased deformability of erythrocytes ex vivo [28]. EVs in allogeneic red blood cell concentrates have been linked to transfusion-related immunomodulation [24,29]. Further research is needed to investigate the clinical relevance of EVs from salvaged red blood cells in not critically ill patients undergoing major orthopedic surgery.

We observed a wide range of erythrocyte EVs (Table 1). Inter-individual variation in EVs has also been found in red blood cell concentrate preparation [25]. In our study, all patient's erythrocytes were collected intra- and postoperatively from the wound for $6 \mathrm{~h}$, dissolved in the same anticoagulated crystalloid solution, with no methodological differences in washing and retransfusion. According to the manufacturer's information, there is a range in centrifugation acceleration from 820 to $1480 \mathrm{G}$. In clinical practice of autologous perioperative cell salvage, there is no monitoring of $G$ during the centrifugation steps and 
G cannot be set by the user. We speculate that different mechanical forces may-at least in part-account for the wide range of erythrocyte EVs (Table 1). Furthermore, patients' erythrocyte susceptibility for microparticle formation may be different (e.g., in pre-existing (subclinical) hematological diseases). Comparing speed and types of centrifugation (continuous versus intermittent), various washing solutions, and salvage duration, investigating time-dependency, the effect of cell salvage processing on erythrocyte EV formation, and short-term storage lesion within $6 \mathrm{~h}$ until retransfusion would be of clinical interest.

Flow cytometry is considered a standard for counting and characterizing cells by their size and surface antigens. Microparticles can also be identified and quantitated by flow cytometry [30]. Standardization of EV analyses of whole blood samples and definitions of normal ranges are warranted. EV assessment could get part of the quality assessment of new extracorporeal devices including cell savers [31,32]. Our aim was, on the one hand, to document the cellular origin of the vesicles as much as possible and, on the other hand, to use the simplest and fastest routinely applicable method that could represent a further step towards standardization.

The discussion of our results with previous work is, for the following reasons, extremely difficult or almost impossible, as the methodologies used differ greatly. In our study we used a highly sensitive flow cytometer with avalanche photodiodes, which shows excellent sensitivity in the red excitation and emission area. Especially for this determination, we used lactadherin, which was conjugated for our purpose with AF647, which can be excited with a $638 \mathrm{~nm}$ laser and has an emission maximum at approx. $670 \mathrm{~nm}$ wavelength. In addition to the detection of phosphatidylserine (unfortunately PS is only about $30-40 \%$ expressed on the "classic" microvesicles), we stained our samples with calcein AM, which was converted to a fluorescent substance by intravesicular esterases. This enabled us to detect the non-PS positive events as well and thus detect a significantly larger number of EVs carrying the cellular specific markers.

\title{
5. Conclusions
}

In conclusion, our experiments indicate that cell salvage using Xtra does not induce relevant amounts of EVs from platelets, myeloid, and monocytic origin. However, autologous cell salvaged blood contains high and variable amounts of erythrocyte EVs.

Author Contributions: Conceptualization, M.K., A.S. and S.K.; methodology, A.S.; formal analysis, M.K., L.S. and A.P.; resources, A.S. and S.K.; data curation, A.S.; writing-original draft preparation, S.K.; writing-review and editing, M.K. and A.S.; visualization, A.S. All authors have read and agreed to the published version of the manuscript.

Funding: This research received no external funding.

Institutional Review Board Statement: The study was conducted according to the guidelines of the Declaration of Helsinki, and approved by the Ethics Committee of Sigmund Freud University Vienna (protocol code 002/2018, 5 June 2018).

Informed Consent Statement: Written informed consent was obtained from all subjects involved in the study. Written informed consent has been obtained from the patient(s) to publish this paper.

Acknowledgments: We would like to thank Dario Fleischmann und Michael Schrödl for administrative assistance and Novomed Handels-GesmbH, Vienna, Austria for donating reagents used for flow cytometric experiments.

Conflicts of Interest: The authors declare no conflict of interest.

\author{
Abbreviations \\ EV Extracellular Vesicle \\ PBM Patient Blood Management \\ RBC Red Blood Cell Concentrate
}




\section{References}

1. Kozek-Langenecker, S.A.; Afshari, A.; Albaladejo, P.; Santullano, C.A.; De Robertis, E.; Filipescu, D.C.; Fries, D.; Goerlinger, K.; Haas, T.; Imberger, G.; et al. Management of severe perioperative bleeding: Guidelines from the European Society of Anesthesiology. Eur. J. Anesthesiol. 2017, 34, 332-395. [CrossRef]

2. Carless, P.A.; Henry, D.A.; Moxey, A.J.; O'Connell, D.; Brown, T.; Fergusson, D.A. Cell salvage for minimising perioperative allogeneic blood transfusion. Cochrane Database Syst. Rev. 2010, 4, CD001888.

3. van Bodegom-Vos, L.; Voorn, V.M.; So-Osman, C.; Vlieland, T.P.; Dahan, A.; Koopman-van Gemert, A.W.; Vehmeijer, S.B.; Nelissen, R.G.; Marang-van de Mheen, P.J. Cell salvage in hip and knee arthroplasty: A meta-analysis of randomized controlled trials. J. Bone Jt. Surg. Am. 2015, 97, 1012-1021. [CrossRef] [PubMed]

4. Xie, J.; Feng, X.; Ma, J.; Kang, P.; Shen, B.; Yang, J.; Zhou, Z.; Pei, F. Is postoperative cell salvage necessary in total hip or knee replacement? A meta-analysis of randomized controlled trials. Int. J. Surg. 2015, 21, 135-144.

5. Dai, B.; Wang, L.; Djaiani, G.; Mazer, C.D. Continuous and discontinuous cell-washing autotransfusion systems. Cardiothorac. Vasc. Anesth. 2004, 18, 210-217. [CrossRef]

6. Donadee, C.; Raat, N.J.; Kanias, T.; Tejero, J.; Lee, J.; Kelley, E.; Zhao, X.; Liu, C.; Reynolds, H.; Azarov, I.; et al. Nitric oxide scavenging by red blood cell microparticles and cell-free hemoglobin as a mechanism for the red cell storage lesion. Circulation 2011, 26, 465-476. [CrossRef] [PubMed]

7. Thery, C.; Ostrowski, M.; Segura, E. Membrane vesicles as conveyors of immune responses. Nat. Rev. Immunol. 2009, 9, 581-593. [CrossRef] [PubMed]

8. Fischer, D.; Büssow, J.; Meybohm, P.; Weber, C.F.; Zacharowski, K.; Urbschat, A.; Müller, M.M.; Jennewein, C. Microparticles from stored blood cells enhance procoagulant and proinflammatory activity. Transfusion 2017, 57, 2701-2711. [CrossRef]

9. Overdevest, E.; Lanen, P.; Feron, J.; van Hees, J.; Tan, M. Clinical evaluation of the Sorin Xtra autotransfusion system. Perfusion 2012, 27, 278-283. [CrossRef] [PubMed]

10. Wisgrill, L.; Lamm, C.; Hartmann, J.; Preißing, F.; Dragosits, K.; Bee, A.; Hell, L.; Thaler, J.; Ay, C.; Pabinger, I.; et al. Peripheral blood microvesicles secretion is influenced by storage time, temperature, and anticoagulants. Cytometry A 2016, 89, 663-672. [CrossRef]

11. Bratton, D.L.; Fadok, V.A.; Richter, D.A.; Kailey, J.M.; Frasch, S.C.; Nakamura, T.; Henson, P.M. Polyamine regulation of plasma membrane phospholipid flip-flop during apoptosis. J. Biol. Chem. 1999, 74, 28113-28120. [CrossRef] [PubMed]

12. Arraud, N.; Gounou, C.; Turpin, D.; Brisson, A.R. Fluorescence triggering: A general strategy for enumerating and phenotyping extracellular vesicles by flow cytometry. Cytometry A 2016, 89, 184-195. [CrossRef] [PubMed]

13. Keuren, J.F.; Magdeleyns, E.J.; Govers-Riemslag, J.W.; Lindhout, T.; Curvers, J. Effects of storage-induced platelet microparticles on the initiation and propagation phase of blood coagulation. Br. J. Haematol. 2006, 134, 307-314. [CrossRef]

14. Kireev, D.A.; Popenko, N.Y.; Pichugin, A.V.; Panteleev, M.A.; Krymskaya, O.V.; Ataullakhanov, F.I.; Sinauridze, E.I. Platelet microparticle membranes have 50- to 100-fold higher specific procoagulant activity than activated platelets. Thromb. Haemost. 2007, 97, 425-434. [CrossRef]

15. Antonova, O.A.; Shustova, O.N.; Golubeva, N.V.; Yakushkin, V.V.; Alchinova, I.B.; Karganov, M.Y.; Mazurov, A.V. Coagulation properties of erythrocyte derived membrane microparticles. Biomed. Khim. 2019, 65, 214-221. [CrossRef]

16. Horstmann, L.L.; McCauley, R.F.; Jy, W.; Ahn, Y.S. Tissue factor-negative cell-derived microparticles play a distinctive role in hemostasis: A viewpoint review. Semin. Thromb. Hemost. 2019, 45, 509-513. [CrossRef] [PubMed]

17. Projahn, D.; Koenen, R.R. Platelets: Key players in vascular inflammation. J. Leukoc. Biol. 2012, 92, 1167-1175. [CrossRef]

18. Biro, E.; van den Goor, J.M.; de Mol, B.A.; Schaap, M.C.; Ko, L.Y.; Sturk, A.; Hack, C.E.; Nieuwland, R. Complement activation on the surface of cell-derived microparticles during cardiac surgery with cardiopulmonary bypass-Is retransfusion of pericardial blood harmful? Perfusion 2011, 26, 21-29. [CrossRef]

19. Kozek-Langenecker, S.; Fenger-Eriksen, C.; Thienpont, E.; Barauskas, G. European guidelines on perioperative venous thrombiembolism prophylaxis. Eur. J. Anaesthesiol. 2017, 34, 1-7.

20. Oswald, E.; Streif, W.; Hermann, M.; Hengster, P.; Mittermayr, M.; Innerhofer, P. Intraoperative salvaged red blood cells contain nearuly no functionally active platelets, but exhibit formation of microparticles: Results of a pilot study in orthopedic patients. Transfusion 2010, 50, 400-406. [CrossRef]

21. Dong, P.; Che, J.; Li, X.; Tian, M.; Smith, F.G. Quick biochemical markers for assessment of quality control of intraoperative cell salvage: A prospective observational study. J. Cardiothorac. Surg. 2014, 9, 86. [CrossRef]

22. Wang, X.; Ji, B.; Zhang, Y.; Zhu, X.; Liu, J.; Long, C.; Zheng, Z. Comparison of the effects of three cell saver devices on erythrocyte function during cardiopulmonary bypass procedure-A pilot study. Artif. Organs 2012, 36, 931-935. [CrossRef]

23. Serrick, C.J.; Scholz, M.; Melo, A.; Singh, O.; Noel, D. Quality of red blood cells using qutotransfusion devices: A comparative analysis. J. Extracorpor. Technol. 2003, 35, 28-34.

24. Almizraq, R.J.; Seghatchian, J.; Acker, J.P. Extracellular Vesicles in Transfusion-Related Immunomodulation and the Role of Blood Component Manufacturing. Transfus. Apher. Sci. 2016, 55, 281-291. [CrossRef] [PubMed]

25. Wannez, A.; Devalet, B.; Chatelain, B.; Chatelain, C.; Dogné, J.-M.; Mullier, F. Extracellular vesicles in red blood cell concentrates: An overview. Transfus. Med. Rev. 2019, 33, 125-130. [CrossRef] [PubMed]

26. Hariri, G.; Bourcier, S.; Marjanovic, Z.; Joffre, J.; Lemarié, J.; Lavillegrand, J.-R.; Charue, D.; Duflot, T.; Bigé, N.; Baudel, J.-L.; et al. Exploring the microvascular impact of red blood cell transfusion in intensive care unit patients. Crit. Care 2019, 23, 292. [CrossRef] 
27. Tissot, J.; Rubin, O.; Canellini, G. Analysis and clinical relevance of microparticles from red blood cells. Curr. Opin. Hematol. 2010, 17, 571-577. [CrossRef]

28. Subramani, K.; Raju, S.P.; Chu, X.; Warren, M.; Pandya, C.D.; Hoda, N.; Fulzele, S.; Raju, R.P. Effect of plasma-derived extracellular vesicles on erythrocyte deformability in polymicrobial sepsis. Int. Immunopharmacol. 2018, 65, 244-247. [CrossRef]

29. Almizraq, R.J.; Norris, P.J.; Inglis, H.; Menocha, S.; Wirtz, M.R.; Juffermans, N.P.; Pandey, S.; Spinella, P.C.; Acker, J.P.; Muszynski, J.A. Blood manufacturing methods affect red blood cell product characteristics and immunomodulatory activity. Blood Adv. 2018, 25, 2296-2306. [CrossRef] [PubMed]

30. Noulsri, E. Quantification of cell-derived microparticles in blood products and its potential application in transfusion laboratories. Lab. Med. 2020, 51, 452-459. [CrossRef] [PubMed]

31. Rogers, S.C.; Iv, F.T.M.; McDonough, R.; Timm, D.D.; Hovmand-Warner, E.; Frazier, E.; Thomas, K.A.; Spinella, P.C.; Doctor, A. Effect of plasma processing and storage on microparticle abundance, nitric oxide scavenging and vasoactivity. Transfusion 2019, 59, 1568-1577. [CrossRef] [PubMed]

32. Seyfried, T.; Breu, A.; Gruber, M.; Reipert, J.; Hansen, E. Processing of small volumes in blood salvage devices. Transfusion 2014, 54, 2775-2781. [CrossRef] [PubMed] 